\title{
Modified spin relaxation mechanism by tunable coupling between interfacial two-dimensional electron gases in correlated oxide heterostructures
}

\author{
M. Huijben, * G. W. J. Hassink, M. P. Stehno, Z. L. Liao, G. Rijnders, A. Brinkman, and G. Koster \\ MESA+ Institute for Nanotechnology, University of Twente, 7500 AE, Enschede, Netherlands
}

(Received 2 February 2017; published 25 August 2017)

\begin{abstract}
Control of spin relaxation is an important prerequisite for the successful implementation of spintronic devices in a materials system. We realized two directly coupled two-dimensional (2D) electron gases (2DEG-2DEG) in a $\mathrm{LaAlO}_{3}-\mathrm{SrTiO}_{3}$ heterostructure system and observed a modification of the spin relaxation mechanism by varying the coupling strength. A strong enhancement of the carrier density for separation distances below a critical thickness of 6 unit cells was revealed. Electric-field-dependent analysis demonstrated tuning from positive to negative magnetoresistance for large separation distances of 10 unit cells indicating Rashba-type spin-orbit coupling, while for small separation distances of only 1 unit cell the magnetoresistance always remained positive. Analysis of the spin-orbit relaxation time and elastic scattering time revealed a modification of the spin relaxation mechanism between Elliott-Yafet and D'yakonov-Perel' for separation distances of 1 and 10 unit cells, respectively. The tunable spin relaxation fits very well with the presence (or absence) of structural inversion symmetry in our coupled 2DEGs system for different separation distances.
\end{abstract}

DOI: 10.1103/PhysRevB.96.075310

\section{INTRODUCTION}

The realization of well-defined quantum wells in layered semiconductor structures has enabled confinement of electrons (and holes) in adjacent two-dimensional (2D) planes. At small separation distances, Coulomb interactions affect interlayer coherence between the charge carriers, leading to new exotic phases, e.g., Bose-Einstein condensates of bilayer excitons [1-4], bilayer fractional quantum Hall states [5], and superfluidity [6].

Quantum-confined electron systems have also been realized in highly ordered epitaxial multilayer systems of correlated oxide materials. An interesting example is the $\mathrm{LaAlO}_{3}$ (LAO)- $\mathrm{SrTiO}_{3}$ (STO) heterostructure system where a twodimensional electron gas forms at the interface between the two band insulators [7]. This materials system has attracted interest for spintronics applications as it features intrinsic ferromagnetism [8-11] as well as gate-tunable spin-orbit interaction [12-16] and spin polarization [17]. Gate-controlled spin injection [18] and spin-to-charge conversion [19,20] have also been demonstrated. An important stepping stone for the successful implementation of spintronics devices is control over the spin relaxation in the material. In this study, we demonstrate a modification between Elliott-Yafet and D'yakonov-Perel' spin-orbit scattering for strongly and weakly coupled LAO/STO 2DEGs, respectively.

Two types of interfaces exist in the LAO/STO materials system, one where LAO grown on $\mathrm{TiO}_{2}$-terminated STO was found to exhibit $n$-type conductivity, whereas the equivalent $p$-type structure, with LAO grown on SrO-terminated STO, remained insulating [7]. Previous studies have also reported the growth and characterization of coupled conducting interfaces in multilayer LAO/STO heterostructures [21,22], where the addition of an extra STO capping layer changes the situation dramatically, preventing structural and chemical

*m.huijben@utwente.nl reconstructions at the LAO surface, and results in metallic behavior below the conventional threshold of 3-4 unit cells [23], even down to a single LAO unit cell layer.

An interesting consequence is the occurrence of a twodimensional hole gas (2DHG) at the STO surface formed by the oxygen $2 p$ states from which electrons have been transferred to the two-dimensional electron gas (2DEG) at the STO-LAO interface underneath. This implies a parallel configuration of a two-dimensional electron conductor and a two-dimensional hole conductor, at a distance of only about 2 perovskite unit cells, which is less than $1.0 \mathrm{~nm}$. Such situation is reminiscent of coupled 2DEG-2DHG systems in semiconductor heterostructures where typical separation distances of several tens of nanometers are present [24]. Evidence for the occurrence of parallel conducting 2D sheets (2DEG and 2DHG) in LAO/STO heterostructures was provided by magnetotransport studies and ultraviolet photoelectron spectroscopy, [22] which demonstrated the presence of electrons as well as holes on a macroscopic level. Subsequent local characterization of the electronic structure of those closely coupled 2DEG and 2DHG interfaces by scanning tunneling microscopy/spectroscopy experiments revealed the individual interface (2DEG) and surface (2DHG) conducting sheets and their temperature-dependent coupling [25]. For the symmetrically related structure, exhibiting two parallel 2D electron gases (2DEG-2DEG), topological superconductivity was predicted for coupled LAO/STO interface systems [26]. A system of closely spaced 2DEG bilayers was studied in LAO/STO superlattices indicating spin-orbit interaction [27]. However, the 2DHG interface, which was still present between the two 2DEG interfaces, was not taken into consideration and very similar transport behavior was observed independent of separation distances.

Here, we report on the growth and electrical characterization of two truly coupled 2D electron gases (2DEG-2DEG) in a LAO/STO heterostructure system, revealing a strong enhancement of carrier density for separation distances below a critical thickness of 6 unit cells. The observed electrical 
behavior is dramatically different from the previously reported coupled oxide 2DEG-2DHG system. Furthermore, electricfield-dependent analysis demonstrates tuning from positive to negative magnetoresistance for large separation distances of 10 unit cells indicating Rashba-type spin-orbit coupling, while for small separation distances of only 1 unit cell the magnetoresistance remains always positive. Analysis of the spin-orbit relaxation time and elastic scattering time reveals a modification of the spin relaxation mechanism between Elliott-Yafet and D'yakonov-Perel' for separation distances of 1 and 10 unit cells (uc), respectively. This fits very well with the presence (or absence) of structural inversion symmetry in our coupled 2DEGs system for different separation distances.

\section{RESULTS AND DISCUSSION}

Pulsed laser deposition (PLD) was used to fabricate two different types of LAO/STO heterostructures in which two coupled interfaces are located at a specific separation distance; see Fig. 1. Heterostructures with two 2D electron gases contain two $n$-type $\mathrm{LaO} / \mathrm{TiO}_{2}$ interfaces, while heterostructures with one $2 \mathrm{D}$ electron gas and another $2 \mathrm{D}$ hole gas contain a $n$-type $\mathrm{LaO} / \mathrm{TiO}_{2}$ interface together with a $p$-type $\mathrm{AlO}_{2} / \mathrm{SrO}$
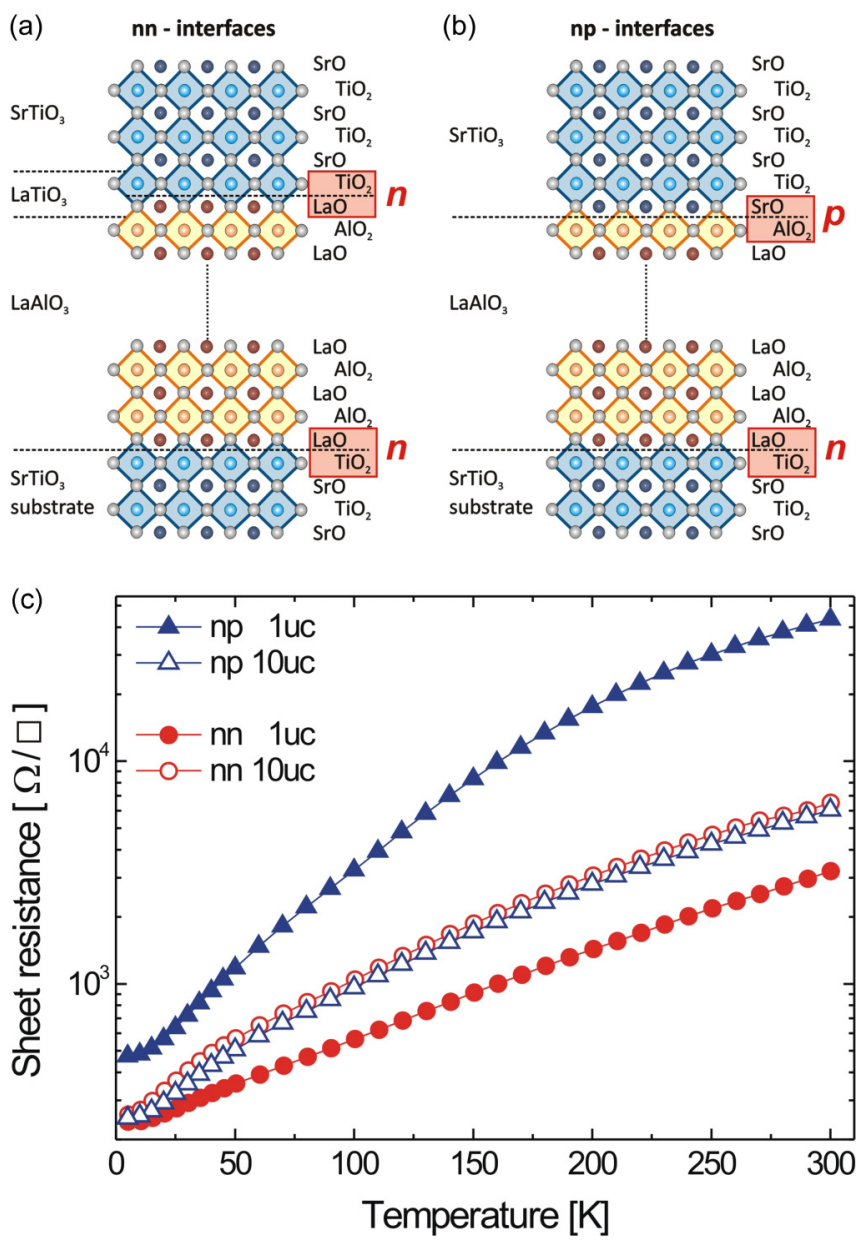

FIG. 1. Interface engineering in LAO/STO heterostructures leading to coupled (a) $n n$ interfaces and (b) $n p$ interfaces, which exhibit different temperature-dependent transport properties (c) for interface separation distances below 10 unit cells. interface. The LAO/STO heterostructures were grown from single-crystalline $\mathrm{LaAlO}_{3}, \mathrm{LaTiO}_{3}$, and $\mathrm{SrTiO}_{3}$ targets by pulsed laser deposition using a $\mathrm{KrF}$ excimer laser operating at $248 \mathrm{~nm}$, while laser fluence and repetition rate were $1.3 \mathrm{~J} / \mathrm{cm}^{2}$ and $1 \mathrm{~Hz}$, respectively. To study the influence of oxygen vacancies both series of $n n$ and $n p$ interfaces were grown at high and low oxygen pressures of $1 \times 10^{-3}$ and $3 \times 10^{-5}$ mbar, respectively, while maintaining a temperature of $850^{\circ} \mathrm{C}$. All heterostructures were grown on $\mathrm{STO}(001)$ substrates, which were chemically treated and annealed to form a $\mathrm{TiO}_{2}$-terminated surface [28]. The bottom $n$-type $\mathrm{LaO} / \mathrm{TiO}_{2}$ interface is equal in all heterostructures and is created by depositing a LAO layer on the $\mathrm{TiO}_{2}$-terminated STO substrate. Due to the epitaxial growth of these two materials, the perovskite atomic stacking is maintained, resulting in a sharp $\mathrm{LaO} / \mathrm{TiO}_{2}$ interface. To form a heterostructure with also a $n$-type interface at the top, the $\mathrm{AlO}_{2}$-terminated surface of the LAO layer is covered with a monolayer of $\mathrm{LaTiO}_{3}$ (LTO) followed by a 20-unit-cell-thick STO layer [see Fig. 1(a)], both to protect the top interface from surface reconstruction and to fully develop the STO band structure. On the other hand, to form a heterostructure with a $p$-type interface at the top, the $\mathrm{AlO}_{2}$-terminated surface of the LAO layer is covered with a thick STO layer; see Fig. 1(b). Data of the latter type of heterostructure have been taken from previous reports [21,22], which were also fabricated by us in the same PLD system. Reflective high-energy electron diffraction (RHEED) during growth indicated layer-by-layer growth for all heterostructures and was used to enable unit-cell-controlled growth.

Both X-ray diffraction (XRD) analysis as well as RHEED confirm the epitaxial growth of all heterostructures through reciprocal space mapping (not shown) by indicating that all heterostructures are fully strained in plane to the STO cubic structure. The coherent growth of all heterostructures is further demonstrated by the observation of a reduced $c$-axis parameter of $3.73 \AA$ for each LAO layer, which is in good agreement with previous reports [29]. The low level of surface roughness was confirmed by atomic force microscopy (AFM) analysis of the surfaces of the LAO/STO heterostructures, indicating smooth terraces separated by clear, single-unit-cell height steps similar to the surface of the initial $\mathrm{TiO}_{2}$-terminated STO (001) substrates.

The transport properties of the LAO/STO heterostructures with $n n$ or $n p$ interfaces exhibit identical temperature dependence for interface separation distances of 10 unit cells (uc); see Fig. 1(c). For smaller separation distances both types of heterostructures maintain metallic behavior, although it can be clearly observed that the changes in temperature dependence show opposite trends for both systems. In contrast to the resistance increase in 1-uc-separated $n p$ interfaces, the $n n$ interfaces with 1-uc separation exhibit a reduction of the resistance. The corresponding carrier density at room temperature depends strongly indeed on the interface separation and shows remarkable differences between the two types of heterostructures with coupled $n n$ or $n p$ interfaces; see Fig. 2(a). Previous studies have demonstrated a decrease in carrier densities for coupled $n p$ interfaces when the separation distance is below $\sim 6$ unit cells [21,22]. Remarkably, coupled $n n$ interfaces show an opposite increase in carrier density for small separation distances. 

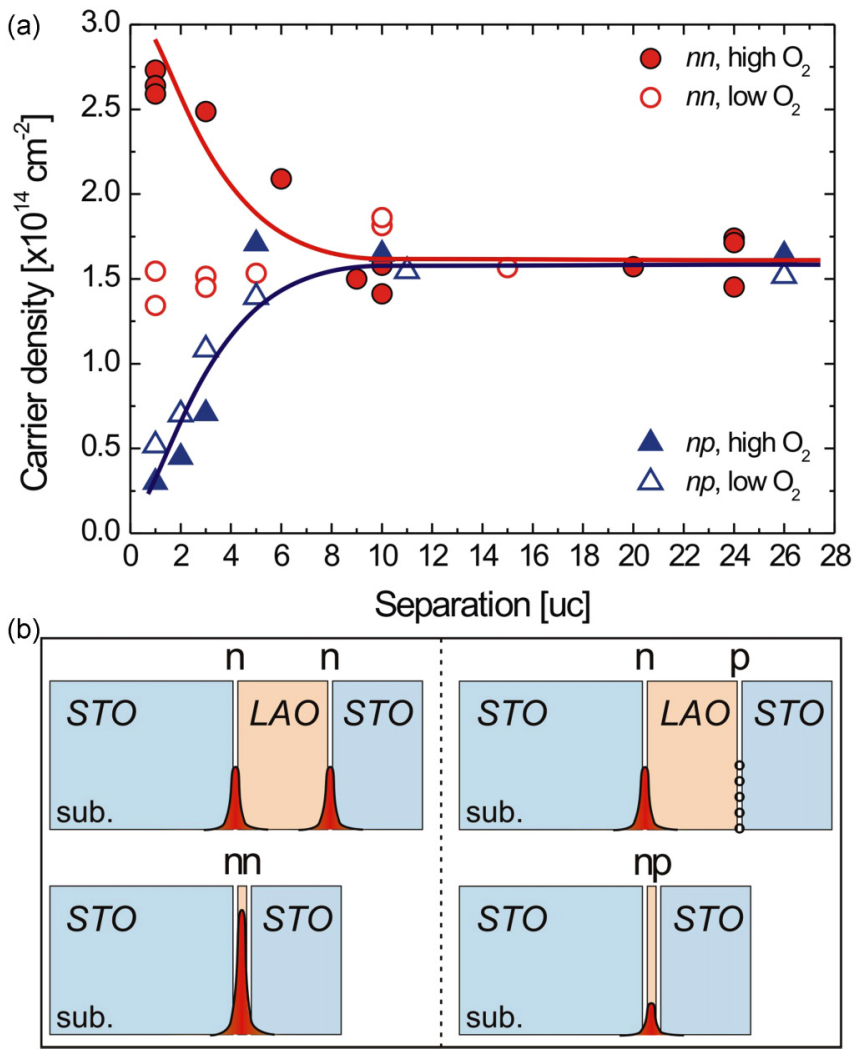

FIG. 2. Separation-distance-dependent transport properties. (a) Carrier density of LAO/STO heterostructures with $n n$ and $n p$ interfaces at $300 \mathrm{~K}$ as a function of the interface separation distance. Heterostructures grown at high and low oxygen pressures, of, respectively, $1 \times 10^{-3}$ and $3 \times 10^{-5} \mathrm{mbar}$, are shown together with guides to the eye. (b) Schematic representations of the carrier distribution at the interfaces for $n n$ and $n p$ interfaces for large and small separation distances.

The observed differences in carrier densities for various LAO layer thicknesses in heterostructures with $n n$ and $n p$ interfaces can be described qualitatively. We assume that 2DEGs of mobile electrons form at the bottom $\mathrm{LaO} / \mathrm{TiO}_{2}$ interfaces in all our heterostructures; see Fig. 2(b). For samples with LAO layers thicker than 6 uc no coupling occurs between the bottom 2DEG and the top LAO/STO interface, resulting in an independent top interface where electrons are generated in the case of a $\mathrm{LaO} / \mathrm{TiO}_{2}$ top interface ( $n n$ interfaces) or hole carriers (i.e., oxygen vacancies) in the case of a $\mathrm{AlO}_{2} / \mathrm{SrO}$ top interface ( $n p$ interfaces). At $300 \mathrm{~K}$, top interface carriers are assumed to have very low mobilities, due to a reduced crystallinity in deposited STO as compared to a STO single-crystal substrate. Therefore, those carriers do not contribute significantly to the Hall signal in the magnetic field range of our measurements. Hence, we only detect mobile carriers at the bottom interfaces with a constant carrier density of $\sim 1.5 \times 10^{14} \mathrm{~cm}^{-2}$ for all LAO thicknesses above $6 \mathrm{uc}$; see Fig. 2(a). However, when the LAO layer thicknesses are reduced below 6 uc coupling occurs between the top and bottom interfaces in both systems. This will result in a reduction of the mobile electrons for $n p$ interfaces as the oxygen vacancies at the top interface will eliminate some electrons at the bottom interface when they are brought into close proximity of each other. Interestingly, for ultrathin LAO layers between $n n$ interfaces the number of mobile electrons is enhanced as the electrons from the top interface apparently are now able to transfer to the high-mobility bottom interface.

Furthermore, the coupled $n p$ interfaces exhibited clear separation distance dependence for low and high oxygen growth pressures, while this effect was only present for coupled $n n$ interfaces grown at high oxygen pressures and absent for low oxygen growth pressures. A possible explanation for these observations is the fact that oxygen vacancies are more easily formed at lower oxygen pressures. In the case of $n p$ interfaces, presumably oxygen vacancies are already formed at the $\mathrm{AlO}_{2} / \mathrm{SrO}$ top interface by the generated holes. Therefore, a lower oxygen growth pressure has a negligible effect and the exhibited LAO thickness dependence is equal for both oxygen pressure regimes. However, in the case of $n n$ interfaces the $\mathrm{LaO} / \mathrm{TiO}_{2}$ top interface is expected to be more sensitive to the formation of oxygen vacancies during growth of a $\mathrm{SrTiO}_{3}$ layer, as compared to extracting oxygen atoms from an already existing crystal structure within the $\mathrm{SrTiO}_{3}$ substrate at the $\mathrm{LaO} / \mathrm{TiO}_{2}$ bottom interface. An enormous amount of oxygen vacancies can be extracted from the $\mathrm{SrTiO}_{3}$ substrate when a single $\mathrm{LAO} / \mathrm{STO}$ interface is grown at $10^{-6} \mathrm{mbar}$, but does not occur at higher growth pressures [8] as used in the present study. The formation of oxygen vacancies at the $\mathrm{LaO} / \mathrm{TiO}_{2}$ top interface could change the details of the conduction bands and/or the generation of electrons. We observe a constant carrier density independent of the LAO layer thickness suggesting the presence of mobile electrons only at the bottom interface.

To reveal the nature of the interfacial charge conduction, we have investigated the temperature-dependent transport properties, in particular, the magnetic field $(B)$ dependence of the sheet resistance $R_{S}(B)$ and Hall resistance $R_{H}(B)$. It was found that $R_{H}(B)$ depends linearly on $B$ above $\sim 100 \mathrm{~K}$ for both systems indicating the presence of a single type of charge carrier. At lower temperatures, the coupled $n p$ interfaces [21] as well as the low-pressure $n n$-interfaces exhibit a linear $R_{H}(B)$ dependence down to $2 \mathrm{~K}$ indicating preservation of a single-band behavior over the complete temperature range. However, the high-pressure $n n$ interfaces clearly show a nonlinearity in the $R_{H}(B)$ dependence below $\sim 100 \mathrm{~K}$ suggesting multiband behavior, similar to previous studies on other types of LAO/STO heterostructures [30,31].

A nonlinear Hall effect can arise from conduction involving different electronic bands and/or spatially separated parallel conducting channels. When only two contributions to conduction are taken into account, $R_{H}(B)$ can be written as $R_{H}(B)=\left[\left(\mu_{1}^{2} n_{1}+\mu_{2}^{2} n_{2}\right)+\left(\mu_{1} \mu_{2} B\right)^{2}\left(n_{1}+\right.\right.$ $\left.\left.\left.n_{2}\right)\right] / e\left(\mu_{1}\left|n_{1}\right|+\mu_{2}\left|n_{2}\right|\right)^{2}+\left(\mu_{1} \mu_{2} B\right)^{2}\left(n_{1}+n_{2}\right)^{2}\right]$. By taking the sheet resistance as a constraint $R_{S}(0)=1 / e\left(n_{1} \mu_{1}+n_{2} \mu_{2}\right)$ all $R_{H}(B)$ data have been fitted in the temperature range from 2 to $100 \mathrm{~K}$. The results of the temperature-dependent fitting parameters are shown in Fig. 3 and indicate a large difference in both the density and the mobility of the majority $\left(n_{1}, \mu_{1}\right)$ and minority $\left(n_{2}, \mu_{2}\right)$ carriers.

The reduction in majority charge carriers for lower temperatures is observed in both $n n$ and $n p$ systems [Figs. 3(a) 

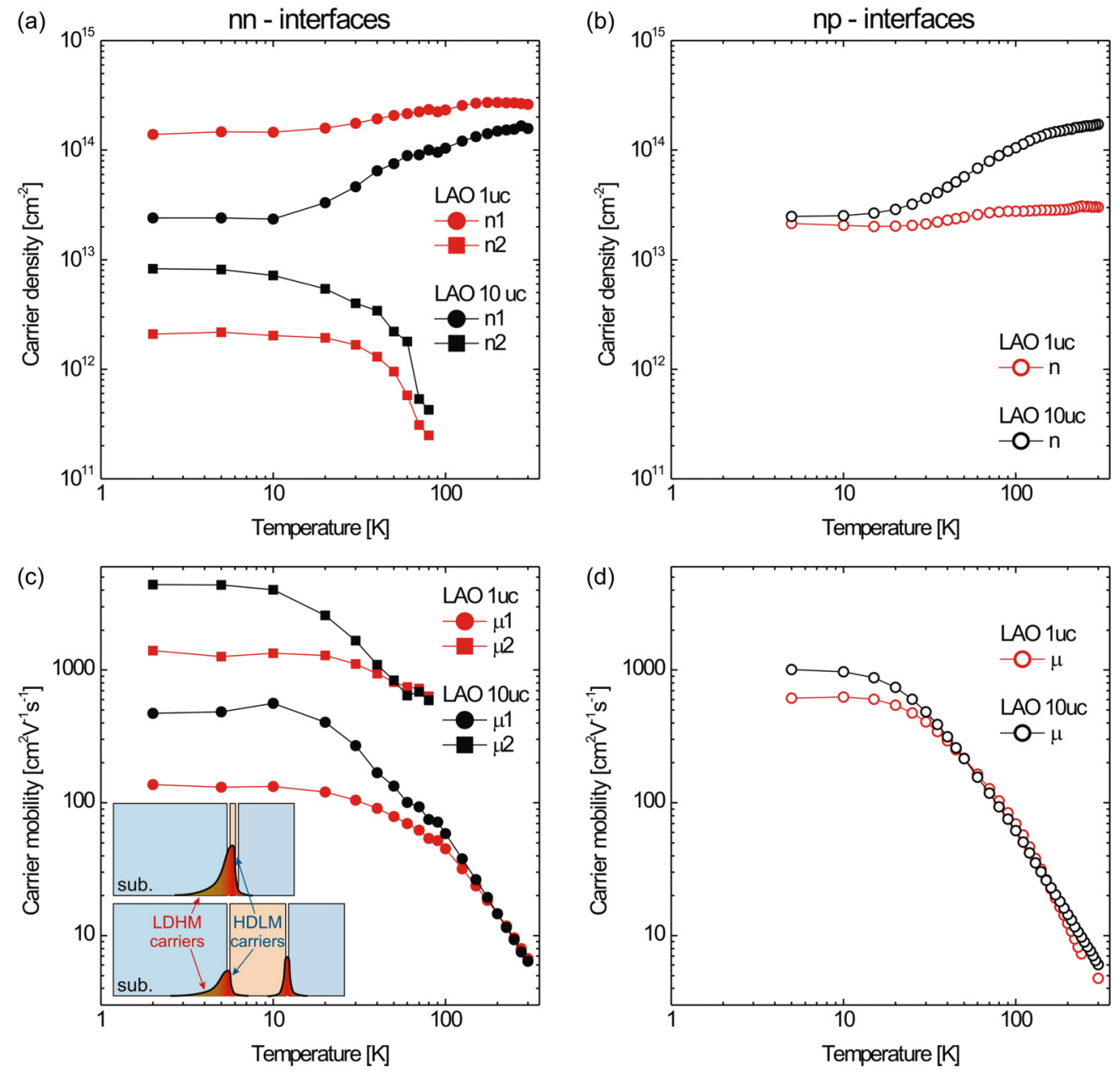

FIG. 3. Temperature-dependent transport properties of $n n$ interfaces (closed symbols) and $n p$ interfaces (open symbols) indicating the carrier density (a,b) and carrier mobility (c,d) of the majority (circles) and minority (squares) carriers for separation distances of 1 uc (red) and $10 \mathrm{uc}$ (black). Inset shows a schematic of the distribution of high-density, low-mobility (HDLM) carriers at the interface as well as low-density, high-mobility (LDHM) carriers away from the interface in the STO substrate for small and large separation distances.

and 3(b)] and indicates the presence of thermally activated carriers with activation energies of $\sim 6 \mathrm{meV}$, reminiscent of local defects [21,32]. The measured carrier density of $\sim 2.5 \times 10^{13} \mathrm{~cm}^{-2}$ at $2 \mathrm{~K}$ for $n p$ interfaces is equal to the majority carrier density $n_{1}$ for $n n$ interfaces with large separation distances of $10 \mathrm{uc}$, suggesting a similar charge conduction of the mobile electrons at the bottom interface. When the separation distance is reduced to $1 \mathrm{uc}$, carrier density $n_{1}$ is increasing over the full temperature range, and carrier density values of $\sim 1.5 \times 10^{14} \mathrm{~cm}^{-2}$ are measured at $2 \mathrm{~K}$. However, the corresponding mobility $\mu_{1}$ of the majority carriers is significantly reduced in closely spaced $n n$ interfaces with values of $\sim 500$ and $\sim 150 \mathrm{~cm}^{2} \mathrm{~V}^{-1} \mathrm{~s}^{-1}$, for 10- and 1-uc distances, respectively, as compared to $\sim 1000$ and $\sim 600 \mathrm{~cm}^{2} \mathrm{~V}^{-1} \mathrm{~s}^{-1}$ for $n p$ interfaces [see Figs. 3(c) and 3(d)]. Interestingly, the generated minority carriers $n_{2}$ for $n n$ interfaces exhibit much larger mobilities $\mu_{2}$ with values of $\sim 5500$ and $\sim 1500 \mathrm{~cm}^{2} \mathrm{~V}^{-1} \mathrm{~s}^{-1}$ for separation distances of 10 and $1 \mathrm{uc}$, respectively. A strong temperature dependence of the minority carrier density is observed to be saturating at low temperatures, which is in good agreement with previous observations in $\mathrm{LaTiO}_{3} / \mathrm{SrTiO}_{3}$ superlattices [33].
The characteristic width of the carrier distribution at the interfaces depends on the strength of the potential screening, i.e., the dielectric permittivity $\varepsilon$. In contrast to the temperature independent $\varepsilon$ of $\sim 25$ for LAO bulk crystals [34], STO exhibits an increase in permittivity by two orders of magnitude when the temperature is lowered, $\varepsilon$ of $\sim 5000$ at $2 \mathrm{~K}$ [35]. With the enhanced $\varepsilon$ at low temperatures, the screening of the electric field from the interfacial carriers becomes more effective and the width of the carrier distribution at the bottom interface is broadened; see inset in Fig. 3(c). This creates high-density, low-mobility (HDLM) carriers at the bottom interface as well as low-density, high-mobility (LDHM) carriers away from the bottom interface in the STO substrate. The increase in the minority carrier density is indeed similar to the temperature dependence of $\varepsilon$ in bulk STO [35]. A similar, permittivitydriven redistribution of carriers was demonstrated in bilayers [36] and superlattices [33].

To elucidate the relationship between the transport properties of mobile carriers and their spatial distribution in coupled $n n$ interfaces, we carried out magnetotransport measurements with an applied back-gate voltage $V_{G}$. The electric field was applied through a back-side contact across the $0.5-\mathrm{mm}$-thick 

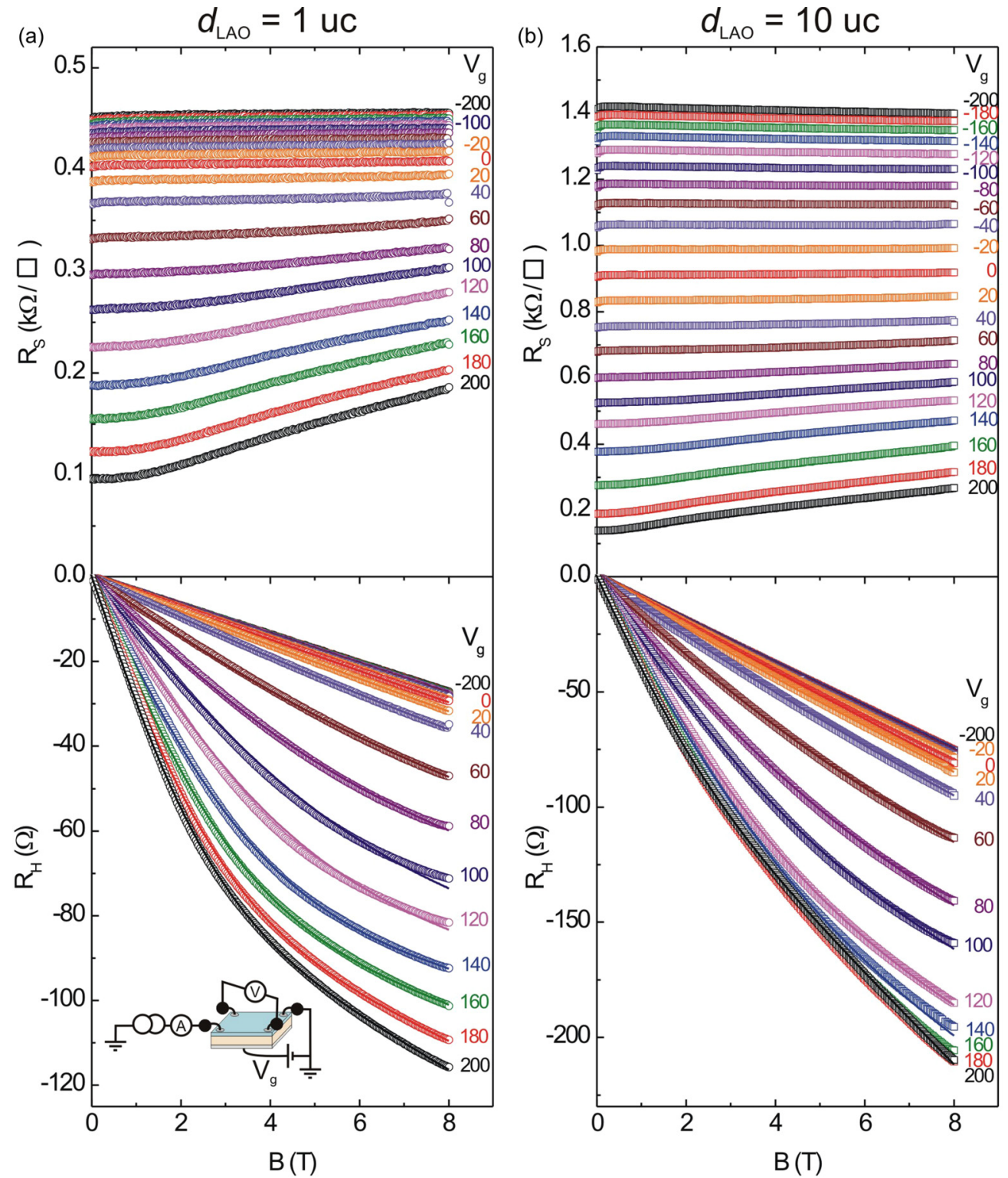

FIG. 4. Magnetic-field-dependent sheet resistance $R_{S}$ (top) and Hall resistance $R_{H}$ (bottom) as a function of gate voltage ( $\left.V_{G}\right)$ at $100 \mathrm{mK}$ for $n n$ interfaces with separation distances of $1 \mathrm{uc}$ (a) and $10 \mathrm{uc}$ (b). $V_{G}$ was varied in steps of $20 \mathrm{~V}$ between -200 and $200 \mathrm{~V}$. Solid lines are fitted curves using the two-channel model. Inset shows a schematic of the experimental setup.

STO substrate; see inset in Fig. 4(a). The measurements were performed at $100 \mathrm{mK}$, down to which no superconductivity was observed. The sheet resistance (top) and Hall resistance (bottom) as a function of magnetic field are shown in Figs. 4(a) and 4(b) for $n n$ interfaces with separation distances of, respectively, 1 and $10 \mathrm{uc}$. The gate voltage was varied in steps of $20 \mathrm{~V}$ between -200 and $200 \mathrm{~V}$. For large positive $V_{G}$ both samples become more conductive, while negative $V_{G}$ correspond to a more resistive state. Furthermore, $R_{H}(B)$ exhibits a linear dependence on magnetic field for negative $V_{G}$, whereas a strong nonlinearity is observed for positive $V_{G}$ biasing. The crossover from linear to nonlinear behavior of $R_{H}(B)$ with increasing $V_{G}$ resembles the behavior for reduced temperatures (Fig. 3). The two-channel model was also applied to fit the $R_{H}(B)$ behavior at different $V_{G}$ and good agreement is found between the experimental data and the solid fitted lines in the bottom figures of Figs. 4(a) and 4(b) for $n n$ interfaces with separation distances of 1 and $10 \mathrm{uc}$, respectively.

The gate voltage dependence of the transport properties for $n n$ interfaces extracted from the fits is presented in Fig. 5, where the strong dependence of sheet resistance on $V_{G}$ for both samples is shown in Fig. 5(a). The density $n_{1}$ of the majority carriers does not depend much on $V_{G}$ below $120 \mathrm{~V}$, although for higher $V_{G}$ a reduction is clearly observed [Fig. 5(b)]. Such decrease is incompatible with a model requiring a fixed electronic band structure, as raising the Fermi energy should always increase the number of available conduction states up to the point where the band is full. Top-gated experiments on a single LAO/STO interface suggested a redistribution of carriers from the $d_{x y}$ band to the $d_{y z, x z}$ bands [37], which indicates that the effective band structure is not fixed, but evolves with gate voltage. Back-side-gated experiments on 
(a)

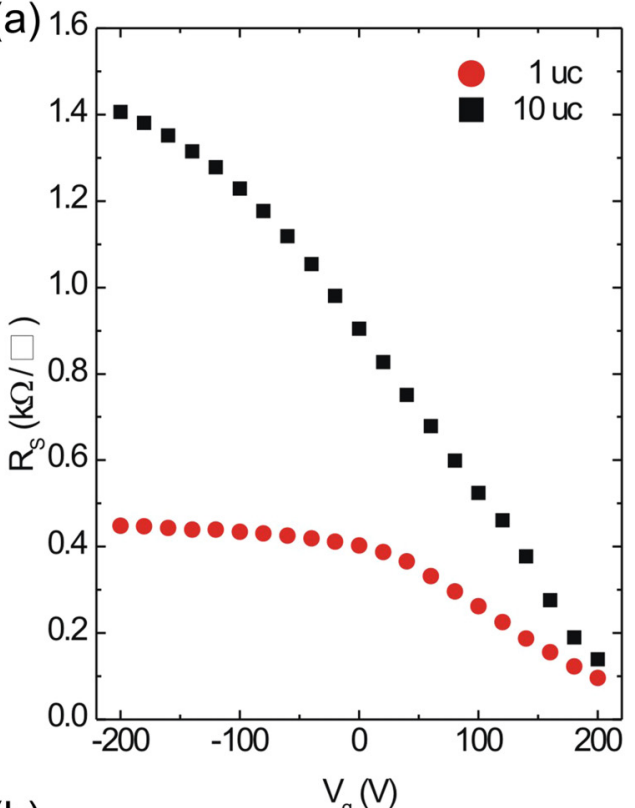

(b)

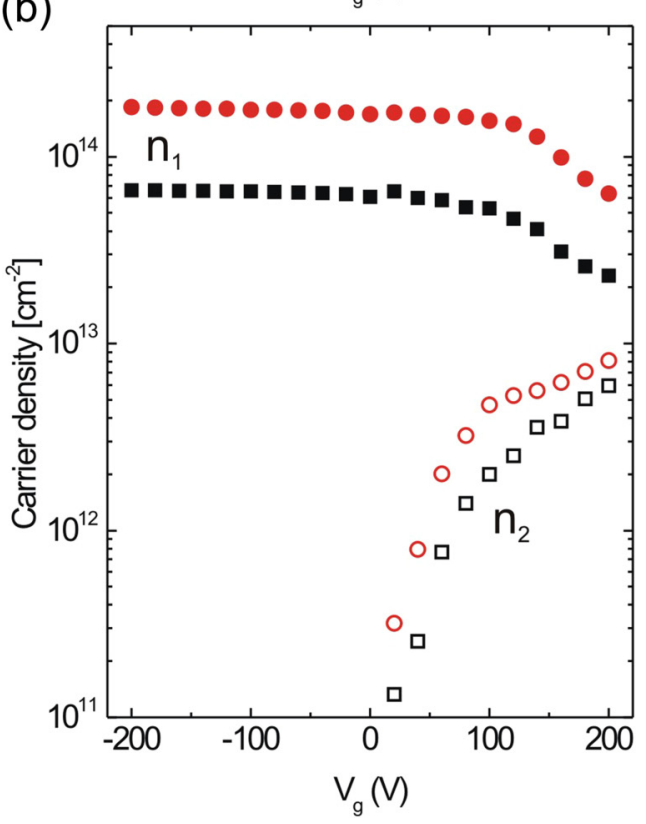

(c)

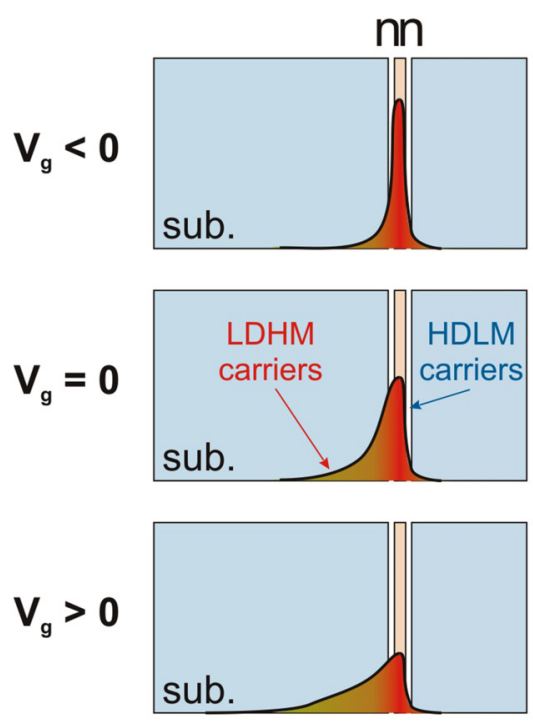

(d)

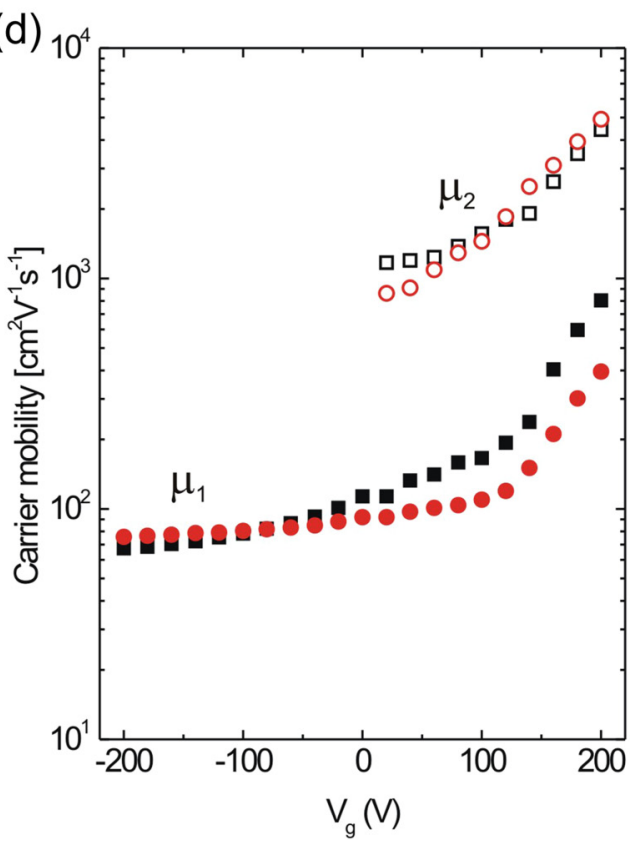

FIG. 5. Gate voltage $\left(V_{G}\right)$-dependent sheet resistance $R_{S}$ (a), carrier density (b), and carrier mobility (d) at $100 \mathrm{mK}$ of the of the majority (closed symbols) and minority (open symbols) carriers for $n n$ interfaces with separation distances of 1 uc (circles) and $10 \mathrm{uc}$ (squares). (c) Schematics of the effects of an external electric field on the carrier distribution.

$\mathrm{LaTiO}_{3} / \mathrm{SrTiO}_{3}$ superlattices demonstrated also a decrease of the total carrier density for increasing bias voltages [33], in good agreement with observations for our $n n$ interfaces. This suggests that changes in the effective band structure with gate voltage also results in variations between mobile and localized charge carriers, where the latter do not contribute to the transport measurements. In contrast, the minority carriers $n_{2}$ exhibit much stronger dependencies on $V_{G}$ as $n_{2}$ increases by two orders of magnitude for high positive $V_{G}$. On the other hand, for negative $V_{G}$ the minority carriers $n_{2}$ become negligible for both samples, very similar to observations for $\mathrm{LaTiO}_{3} / \mathrm{SrTiO}_{3}$ superlattices [33]. The origin of this behavior was understood by taking into account two factors. First, the dielectric permittivity $\varepsilon$ of STO is known to decrease in an external electric field and, therefore, reverses the increase in $\varepsilon$ at low temperatures noted above, thus eliminating the minority carriers $n_{2}$ for negative $V_{G}$. For positive $V_{G}$ this effect is offset by a second factor, which is the flattening of the potential profile toward the gate electrode, as illustrated in Fig. 5(c). The shallower potential profile shifts mobile carriers away from scattering centers at the interface, thereby enhancing the density of the minority carriers with high mobility (LDHM) and reducing the density of the majority carriers with low mobility (HDLM); see Fig. 5(b). However, in the recent study on a single 2DEG at the LAO/STO interface electron-electron correlations had to be considered to explain the gate voltage dependence of the majority and minority carriers [37]. Furthermore, in our coupled $n n$ interfaces the 

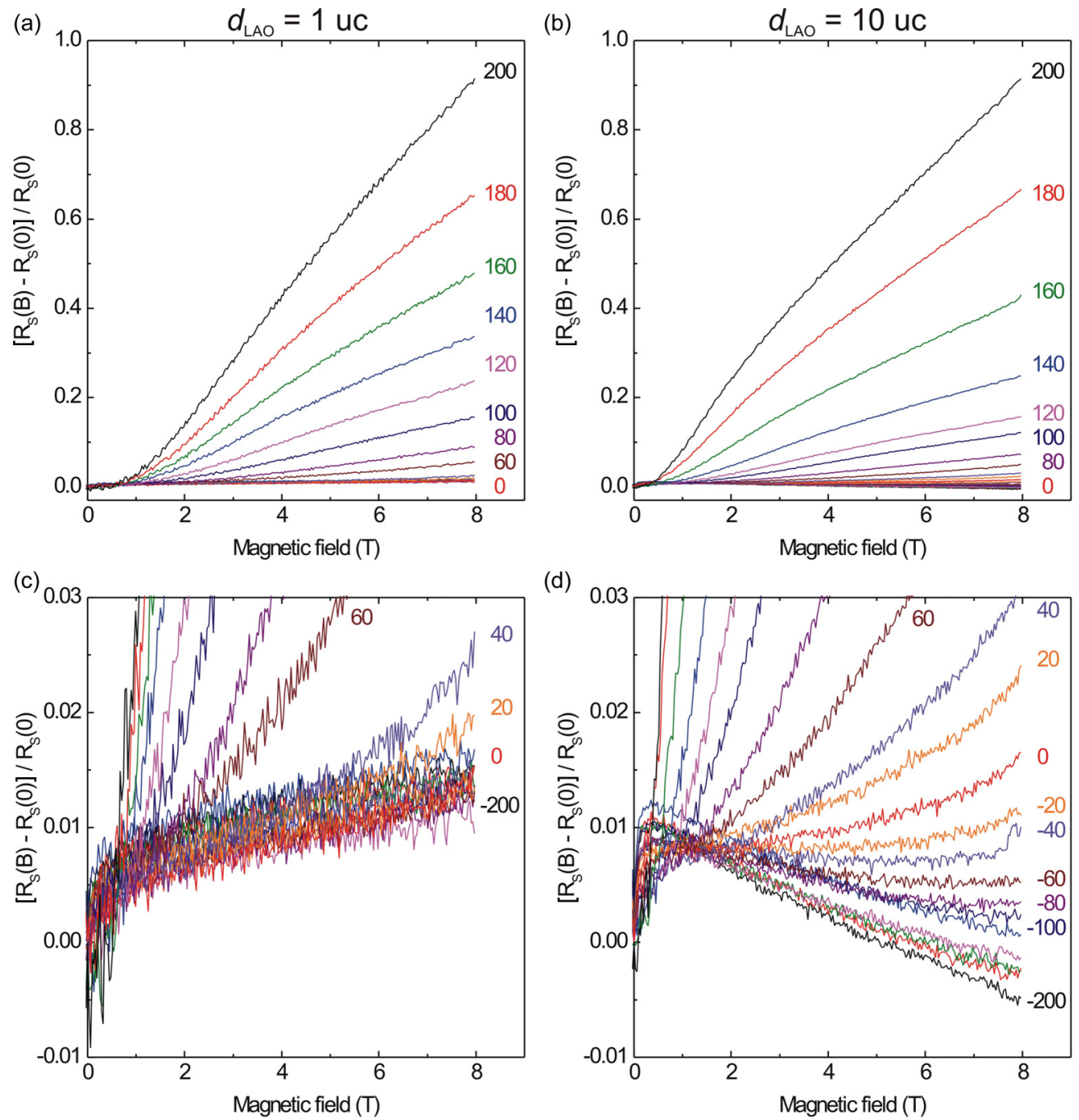

(d)

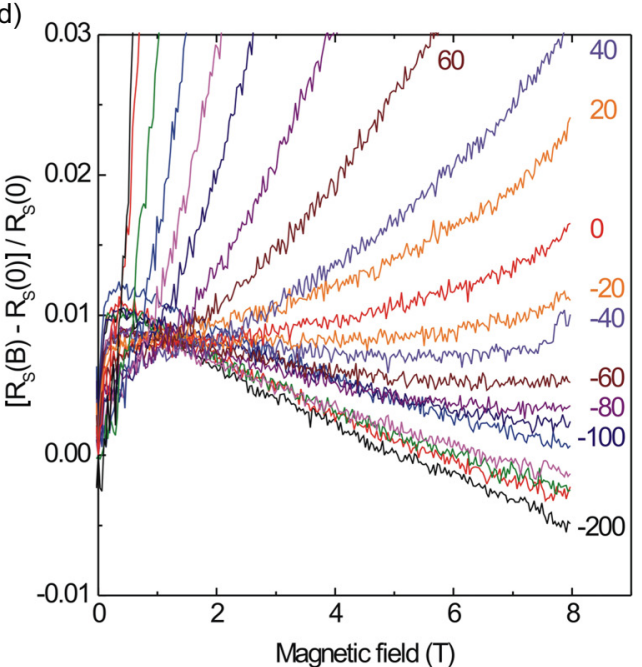

FIG. 6. Magnetic field dependence of normalized sheet resistance $\left[R_{S}(B)-R_{S}(0)\right] / R_{S}(0)$ as a function of gate voltage $\left(V_{G}\right)$ at $100 \mathrm{mK}$ for $n n$ interfaces with separation distances of $1 \mathrm{uc}(\mathrm{a}, \mathrm{c})$ and $10 \mathrm{uc}(\mathrm{b}, \mathrm{d})$.

mobility of both minority and majority carriers is enhanced significantly for large positive $V_{G}$ [Fig. 5(d)].

These results clearly demonstrate the presence of twochannel transport in $n n$ interfaces with separation distances of $1 \mathrm{uc}$ as well as $10 \mathrm{uc}$, which could be caused by conduction involving different electronic bands and/or spatially separated parallel conducting channels. The importance of the spatial separation of such coupled bilayer electron system was studied by the electric-field-dependent magnetoresistance. The normalized sheet resistance $\left[R_{S}(B)-R_{S}(0)\right] / R_{S}(0)$ exhibits a strong positive magnetoresistance for large positive $V_{G}$, which is very similar for $n n$ interfaces with separation distances of $1 \mathrm{uc}$ as well as 10 uc; see Figs. 6(a) and 6(b). In contrast, for negative $V_{G}$ the observed magnetoresistance is minimal in both samples; see Figs. 6(c) and 6(d). For $n n$ interfaces with larger separation distances of $10 \mathrm{uc}$ an additional negative magnetoresistance contribution is observed when $V_{G}$ is lowered from 0 down to $-200 \mathrm{~V}$. Such tuning from positive to negative magnetoresistance by varying the gate voltage is a clear signature of Rashba-type spin-orbit coupling, as previously demonstrated for single-2DEG LAO/STO interfaces
[12,38]. This similarity agrees very well with our previous experimental observations of similar transport behavior for $n n$ and $n p$ interfaces with large separation distances [as shown in Fig. 1(c)] indicating the dominating contribution of the single bottom $n$ interface. The tunability of the Rashba spin-orbit interaction, arising from the breaking of structural inversion symmetry, is substantial; however, no superconductivity was determined down to $15 \mathrm{mK}$ base temperature, in strong contrast to such experiments on single-2DEG LAO/STO interfaces $[12,16]$ and theoretical predictions on bilayer-2DEG-2DEG systems [26].

Interestingly, for $n n$ interfaces with a small separation distance of only 1 uc the magnetoresistance remains positive with negligible changes when $V_{G}$ is lowered from 0 down to $-200 \mathrm{~V}$. Such oxide heterostructures with a very thin $\mathrm{LaO}-\mathrm{AlO}_{2}-\mathrm{LaO}$ layer in between two $\mathrm{SrTiO}_{3}$ layers exhibit a symmetric interface region, considering the spatial extent of the well, which is very different from $\mathrm{LaAlO}_{3}-\mathrm{SrTiO}_{3}$ interfaces with thick $(\sim 10 \mathrm{uc}) \mathrm{LaAlO}_{3}$ layers with clear separation between the 2DEGs. The inversion symmetry of the electron distribution has a dramatic effect on the 
spin precession in a $2 \mathrm{DEG}$ confined at an interface. The accumulation of electrons at a 2DEG interface generates a strong local electric field perpendicular to the conduction plane of the electrons, which translates into a magnetic field in their rest frame. The coupling of the electrons to this field can give rise to a Rashba spin-orbit coupling, whose strength is directly proportional to the interfacial electric field. A more symmetric charge distribution in the sample with 1 uc separation, then, leads to a different spin-orbit strength compared to the case of widely separated interfaces. The well separation thus also tunes the spin-orbit scattering. To reveal this mechanism, we manipulate the interfacial electric fields by an external gate voltage.

The reduction of the spin coherence by the spin-orbit coupling is characterized by the spin relaxation mechanism. The spin-orbit relaxation time $\tau_{s o}$ is an essential ingredient to describe transport in a $2 \mathrm{DEG}$ in the presence of a strong electric field. The two most widely applied mechanisms of spin relaxation are Elliott-Yafet (EY) and D'yakonov-Perel' (DP), which have their roots in metal and semiconductor spintronics. Both mechanisms rely on spin-orbit coupling and momentum scattering, but their effect is opposite to each other. The EY mechanism explains the spin relaxation by spin flips during scattering for systems exhibiting space inversion symmetry. In the presence of spin-orbit coupling Bloch states are an admixture of the spin-up and spin-down states, as interaction of the lattice ions with the conduction electrons, by phonon scattering or spin-conserving impurity, can induce a spin flip. In the case of the EY mechanism a direct proportionality between the spin-orbit relaxation time and the elastic scattering time is predicted, $\tau_{s o} \sim \tau_{e l}$. For the DP mechanism the spins precess between the scattering events. In the absence of space inversion symmetry, the spin-orbit coupling is manifested as a spin-orbit field, e.g., Rashba-type, along which the electron spin precesses. As the electron scatters, the orientation and/or value of the effective magnetic field changes and the electron spin precesses in the randomly spin-orbit field. In the case of the DP mechanism an inverse proportionality exists between the spin-orbit relaxation time and the elastic scattering time, $\tau_{s o} \sim \tau_{e l}^{-1}$. Interestingly, previous studies have reported the interplay between these two mechanisms [39] and demonstrated the unification of both mechanisms within a single theoretical framework [40].

The influence of the spin-orbit interaction, and the corresponding dominant spin-relaxation mechanism, can be assessed by measuring the magnetoconductivity $\Delta \sigma(B)=$ $\sigma(B)-s(0)$ for different gate voltages. The experimental data were fitted with the Maekawa-Fukuyama formula valid in the diffusive regime that describes the change in the conductivity with magnetic field with negligible Zeeman splitting [41],

$$
\begin{aligned}
\frac{\Delta \sigma}{\sigma_{0}}= & -\Psi\left(\frac{1}{2}+\frac{B_{e l}}{B}\right)+\frac{3}{2} \Psi\left(\frac{1}{2}+\frac{B_{i n}+B_{s o}}{B}\right) \\
& -\frac{1}{2} \Psi\left(\frac{1}{2}+\frac{B_{i n}}{B}\right)-\mid \ln \left(\frac{B_{\text {in }}+B_{\text {so }}}{B_{\text {el }}}\right) \\
& +\frac{1}{2} \ln \left(\frac{B_{\text {in }}+B_{\text {so }}}{B_{\text {in }}}\right) \mid-A_{k} \frac{\sigma(0)}{\sigma_{0}} B^{2},
\end{aligned}
$$

where $\Psi$ is the digamma function, $\sigma_{0}=e^{2} / \pi h$ and the parameters $B_{e l}, B_{i n}, B_{s o}$ are the effective fields related to the elastic, inelastic, and spin-orbit relaxation times, respectively. To account for the magnetoconductance at large positive gate voltages, a $B^{2}$ term was added with a Kohler coefficient $A_{k}$ which increases quadratically with the mobility and is indicative of the increasing contribution of a second band of charge carriers to the system [Fig. 5(b)]. Good agreement was obtained between the Maekawa-Fukuyama theory and our experimental data, as shown in Fig. 7.

The evolution of the fitting parameters as a function of the gate voltage is given in Figs. 7(a) and 7(b) for $n n$ interfaces with separation distances of 1 and $10 \mathrm{uc}$, respectively. $B_{i n}$ exhibits only minor variation over the whole range of gate voltages, and the values for $B_{i n}$ at $100 \mathrm{mK}$ are in good agreement with the temperature dependence $B_{i n} \sim T$ observed for a single $2 \mathrm{DEG}$ at the $\mathrm{LAO} / \mathrm{STO}$ interface in a previous study for the temperature range 3-10 K [15], indicating that the inelastic scattering is dominated by the electron-electron interactions [42].

The strongest contribution of all three field components $B_{s o}, B_{e l}, B_{i n}$ was determined when a gate voltage of $-200 \mathrm{~V}$ was applied to the system with $n n$ interfaces at a separation distance of $10 \mathrm{uc}$; see Figs. 7(b) and 7(d). This maximum value of $B_{e l}$ was used in combination with the 2D transport relation $B_{e l}=\hbar / 4 e D_{e l} \tau_{e l}=e / 2 \mu^{2} \hbar^{2} k^{2}$ to derive the elastic field contributions for all gate voltages for $n n$ interfaces with separation distances of 1 and $10 \mathrm{uc}$ by taking into account the mobility values $\mu_{1}$ for the majority carriers; see Fig. 5(d). Although the carrier densities $n_{1}$ were rather constant over a large gate voltage range, the different values for $n n$ interfaces with separation distances of 1 and $10 \mathrm{uc}$, respectively, $18.4 \times$ $10^{13} \mathrm{~cm}^{-2}$ and $6.6 \times 10^{13} \mathrm{~cm}^{-2}$ [see Fig. 5(c)] were taken into account when calculating $\mathrm{k}\left(=\sqrt{2 \pi n_{1}}\right)$ for both systems. For gate voltages of $-80 \mathrm{~V}$ both systems exhibited equal carrier mobilities $\mu_{1}$ [Fig. 5(d)]; therefore, the factor of 3 difference in carrier density $n_{1}$ resulted for this specific gate voltage in a factor of 3 enhancement of $B_{e l}$ for $n n$ interfaces with separation distances of $10 \mathrm{uc}$ as compared to a system with separation distances of $1 \mathrm{uc}$. Interestingly, the spin-orbit field $B_{s o}$ contribution is very similar for both systems with $n n$ interfaces at separation distances of 1 or $10 \mathrm{uc}$. For large negative gate voltages $B_{s o}$ is increasing, in strong contrast to the opposite observation for single-2DEG systems $[12,15]$. However, for all studies an increasing $B_{s o}$ coincides with an increasing carrier density, although $n_{1}$ variation is very small $(\sim 8 \%)$ in our case for negative $V_{G}$. The Kohler coefficient $A_{k}$ remained very small for negative $V_{G}$, where a single-band system with only majority carriers $n_{1}$ was present; see Fig. 5(c). The additional minority carriers $n_{2}$ created a two-band system in which a dramatic $A_{k}$ contribution to the fitting was required for large, positive gate voltages.

To study the spin relaxation mechanism in our coupled $n n$-interface systems, the spin-orbit relaxation time $\tau_{s o}$ and the elastic scattering time $\tau_{e l}$ were determined by the expressions $\tau_{s o}=\hbar / 4 D e B_{s o}$ and $\tau_{e l}=\hbar / 4 D e B_{e l}$, where $D\left(=0.5 v_{f}^{2} \tau_{e l}\right)$ is the diffusion coefficient for $2 \mathrm{D}$ transport and $v_{f}$ is the Fermi velocity. To determine $v_{f}\left(=\hbar k / m^{*}\right)$ an effective mass $m^{*}=3 m_{e}$ was used ( $m_{e}$ is the bare electron mass), taking 

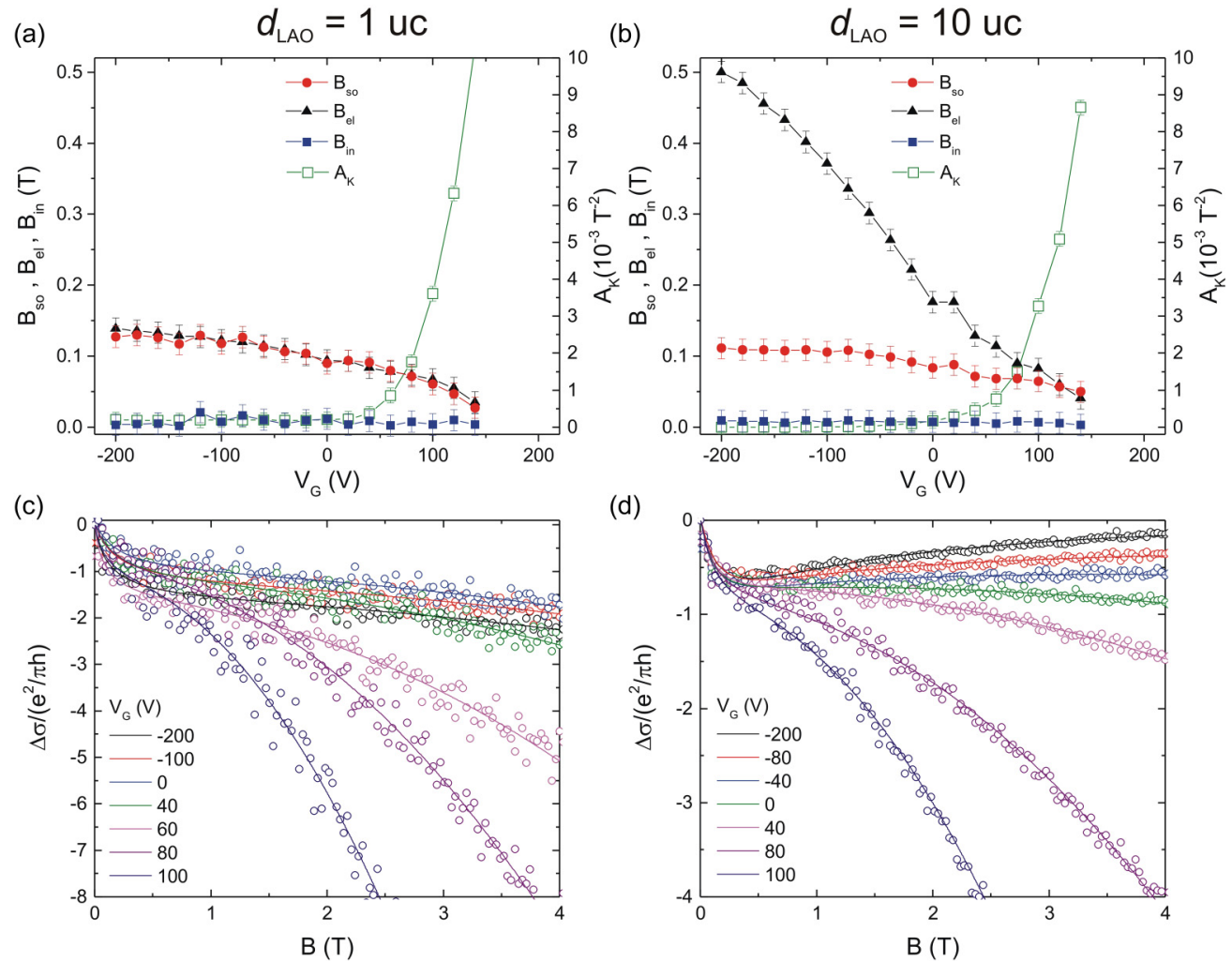

FIG. 7. Analysis of magnetoconductivity at $100 \mathrm{mK}$ for $n n$ interfaces with separation distances of $1 \mathrm{uc}(\mathrm{a}, \mathrm{c})$ and $10 \mathrm{uc}(\mathrm{b}, \mathrm{d})$. (a,b) Evolution of the fitting parameters $B_{s o}, B_{e l}, B_{i n}$, and $A_{k}$ as a function of gate voltage $\left(V_{G}\right)$. (c,d) Best fits according to the Maekawa-Fukuyama theory of the variation of conductance $\Delta \sigma$, normalized with respect to $e^{2} / \pi h$, for different gate voltages.

an average for contributions of the light and heavy bands [43]. Furthermore, the different carrier density values for $n n$ interfaces with separation distances of 1 and $10 \mathrm{uc}$ were taken into account when calculating $k$ for both systems. This results in the following expressions:

$$
\tau_{e l}=\sqrt{\frac{m^{* 2}}{2 e \hbar k^{2}} \frac{1}{B_{e l}}} \text { and } \tau_{s o}=\frac{m^{* 2}}{2 e \hbar k^{2}} \frac{1}{\tau_{e l}} \frac{1}{B_{s o}} .
$$

The gate voltage dependence of the spin-orbit relaxation time $\left(\tau_{s o}\right)$ and the elastic scattering time $\left(\tau_{e l}\right)$ at $100 \mathrm{mK}$ for

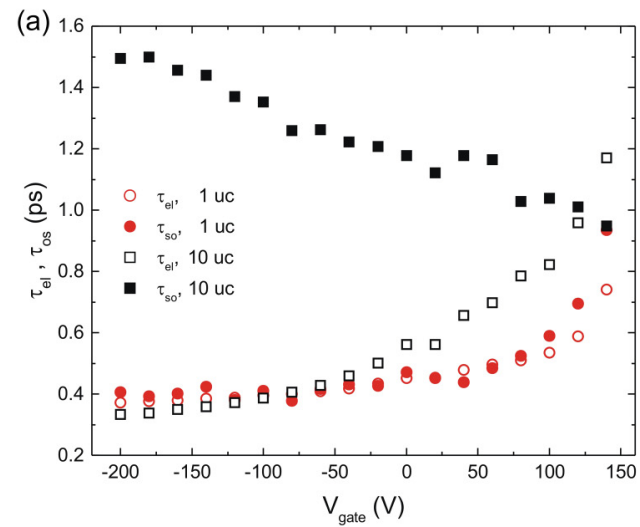

$n n$ interfaces with separation distances of 1 and $10 \mathrm{uc}$ are shown in Fig. 8(a). The variation in $\tau_{e l}$ is very similar for both systems with a rather constant value of $\sim 0.4 \mathrm{ps}$ for negative gate voltages. However, the spin-orbit relaxation time $\left(\tau_{s o}\right)$ displays a very different trend for both systems. Although $n n$ interfaces with a separation distance of $1 \mathrm{uc}$ exhibit $\tau_{\text {so }}$ values very close to $\tau_{e l}, n n$ interfaces with a separation distance of 10 uc give the opposite, increasing trend for negative voltages up to $\sim 1.5 \mathrm{ps}$ ps for $-200 \mathrm{~V}$. When the $\tau_{s o}$ vs $\tau_{e l}$ is plotted, see Fig. 8(b), it is clear that results of the $n n$ interfaces with a separation distance of 10 uc show consistency with the

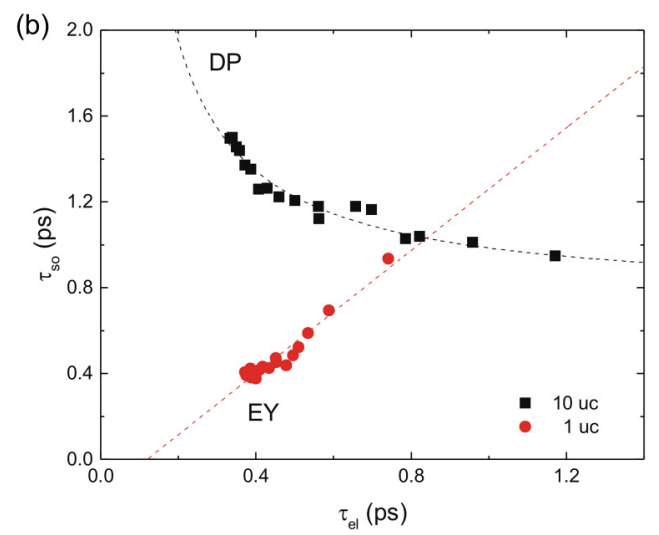

FIG. 8. Analysis of spin relaxation mechanism. (a) Gate voltage dependence of spin-orbit relaxation time $\left(\tau_{s o}\right)$ and elastic scattering time $\left(\tau_{e l}\right)$ at $100 \mathrm{mK}$. (b) $\tau_{s o}$ vs $\tau_{e l}$ showing consistency with Elliott-Yafet (EY) and D'yakonov-Perel' (DP) mechanisms for $n n$ interfaces with separation distances of, respectively, 1 and 10 uc. 
D'yakonov-Perel' (DP) mechanism, in good agreement with previous studies on single 2DEGs $[12,15]$. Interestingly, the results of the $n n$ interfaces with a separation distances of $1 \mathrm{uc}$ display a clear linear relation indicating the presence of the Elliott-Yafet (EY) mechanism.

\section{CONCLUSION}

In conclusion, the modification of the spin-orbit relaxation mechanism fits very well with our hypothesis of the absence (or presence) of structural inversion symmetry in our $n n$ interfaces system for different separation distances. Our experimental observations, indicating the dominating contribution of the single bottom $n$ interface when the separation distance between the two 2 DEGs is large $(\sim 10 \mathrm{uc})$, confirm that no coupling exists with the top interface, leading to equal transport properties for such $n n$ or $n p$ interface system. Therefore, the $2 \mathrm{DEG}$ is confined at the interface between $\mathrm{SrTiO}_{3}$ and $\mathrm{LaAlO}_{3}$, which results in breaking of the structural inversion symmetry and subsequently the existence of the D'yakonov-Perel' spin relaxation mechanism. However, transport properties of closely spaced 2DEGs in a $n n$-interface system for very small separation distances $(\sim 1 \mathrm{uc})$ show coupling between the two 2DEGs indicating that confinement within the complete $\mathrm{SrTiO}_{3}-\mathrm{LaAlO}_{3}-\mathrm{SrTiO}_{3}$ region has to be taken into account. Consequently, structural inversion symmetry is present for this coupled interface region leading to the Elliott-Yafet spin relaxation mechanism. This remarkable modification of the spin relaxation mechanism in complex oxide heterostructures should stimulate further theoretical and experimental investigations, as integration with fully spin-polarized materials could lead to alternative spintronic devices.

\section{ACKNOWLEDGMENTS}

The authors acknowledge funding from the DESCO program of the Dutch Foundation for Fundamental Research on Matter (FOM) with financial support from the Netherlands Organization for Scientific Research (NWO).
[1] J. P. Eisenstein and A. H. MacDonald, Nature 432, 691 (2004).

[2] I. B. Spielman, J. P. Eisenstein, L. N. Pfeiffer, and K. W. West, Phys. Rev. Lett. 84, 5808 (2000).

[3] M. Kellogg, J. P. Eisenstein, L. N. Pfeiffer, and K. W. West, Phys. Rev. Lett. 93, 036801 (2004).

[4] E. Tutuc, M. Shayegan, and D. A. Huse, Phys. Rev. Lett. 93, 036802 (2004).

[5] J. P. Eisenstein, G. S. Boebinger, L. N. Pfeiffer, K. W. West, and S. He, Phys. Rev. Lett. 68, 1383 (1992).

[6] X.-G. Wen and A. Zee, Phys. Rev. Lett. 69, 1811 (1992).

[7] A. Ohtomo and H. Y. Hwang, Nature 427, 423 (2004).

[8] A. Brinkman, M. Huijben, M. Van Zalk, J. Huijben, U. Zeitler, J. C. Maan, W. G. Van der Wiel, G. Rijnders, D. H. A. Blank, and H. Hilgenkamp, Nat. Mater. 6, 493 (2007).

[9] B. Kalisky, J. A. Bert, B. B. Klopfer, C. Bell, H. K. Sato, M. Hosoda, Y. Hikita, H. Y. Hwang, and K. A. Moler, Nat. Commun. 3, 922 (2012).

[10] L. Li, C. Richter, J. Mannhart, and R. C. Ashoori, Nat. Phys. 7, $762(2011)$.

[11] F. Bi, M. Huang, S. Ryu, H. Lee, C. Bark, C. Eom, P. Irvin, and J. Levy, Nat. Commun. 5, 5019 (2014).

[12] A. D. Caviglia, M. Gabay, S. Gariglio, N. Reyren, C. Cancellieri, and J.-M. Triscone, Phys. Rev. Lett. 104, 126803 (2010).

[13] M. Ben Shalom, M. Sachs, D. Rakhmilevitch, A. Palevski, and Y. Dagan, Phys. Rev. Lett. 104, 126802 (2010).

[14] A. Joshua, S. Pecker, J. Ruhman, E. Altman, and S. Ilani, Nat. Commun. 3, 1129 (2012).

[15] S. Hurand, A. Jouan, C. Feuillet-Palma, G. Singh, J. Biscaras, E. Lesne, N. Reyren, A. Barthlmy, M. Bibes, J. Villegas, C. Ulysse, X. Lafosse, M. Pannetier-Lecoeur, S. Caprara, M. Grilli, J. Lesueur, and N. Bergeal, Sci. Rep. 5, 12751 (2015).

[16] G. Herranz, G. Singh, N. Bergeal, A. Jouan, J. Lesueur, J. Gazquez, M. Varela, M. Scigaj, N. Dix, F. Sanchez, and J. Fontcuberta, Nat. Commun. 6, 6028 (2015).

[17] A. Joshua, J. Ruhman, S. Pecker, E. Altman, and S. Ilani, Proc. Natl. Acad. Sci. USA 110, 9633 (2013).
[18] N. Reyren, M. Bibes, E. Lesne, J.-M. George, C. Deranlot, S. Collin, A. Barthelemy, and H. Jaffrès, Phys. Rev. Lett. 108, 186802 (2012).

[19] E. Lesne, Y. Fu, S. Oyarzun, J. C. Rojas-Snchez, D. C. Vaz, H. Naganuma, G. Sicoli, J.-P. Attan, M. Jamet, E. Jacquet, J.-M. George, A. Barthlmy, H. Jaffrès, A. Fert, M. Bibes, and L. Vila, Nat. Mater. 15, 1261 (2016).

[20] J.-Y. Chauleau, M. Boselli, S. Gariglio, R. Weil, G. de Loubens, J.-M. Triscone, and M. Viret, Europhys. Lett. 116, 17006 (2016).

[21] M. Huijben, G. Rijnders, D. H. A. Blank, S. Bals, S. Van Aert, J. Verbeeck, G. Van Tendeloo, A. Brinkman, and H. Hilgenkamp, Nat. Mater. 5, 556 (2006).

[22] R. Pentcheva, M. Huijben, K. Otte, W. E. Pickett, J. E. Kleibeuker, J. Huijben, H. Boschker, D. Kockmann, W. Siemons, G. Koster, H. J. W. Zandvliet, G. Rijnders, D. H. A. Blank, H. Hilgenkamp, and A. Brinkman, Phys. Rev. Lett. 104, 166804 (2010).

[23] S. Thiel, G. Hammerl, A. Schmehl, C. W. Schneider, and J. Mannhart, Science 313, 1942 (2006).

[24] M. Kellogg, J. P. Eisenstein, L. N. Pfeiffer, and K. W. West, Phys. Rev. Lett. 90, 246801 (2003).

[25] M. Huijben, D. Kockmann, J. Huijben, J. E. Kleibeuker, A. van Houselt, G. Koster, D. H. A. Blank, H. Hilgenkamp, G. Rijnders, A. Brinkman, and H. J. W. Zandvliet, Phys. Rev. B 86, 035140 (2012).

[26] S. Nakosai, Y. Tanaka, and N. Nagaosa, Phys. Rev. Lett. 108, 147003 (2012).

[27] H. Ma, Z. Huang, W. Lu, A. Annadi, S. Zeng, L.Wong, S. Wang, T. Venkatesan, and Ariando, Appl. Phys. Lett. 105, 011603 (2014).

[28] G. Koster, B. L. Kropman, A. J. H. M. Rijnders, D. H. A. Blank, and H. Rogalla, Appl. Phys. Lett. 73, 2920 (1998).

[29] M. Huijben, A. Brinkman, G. Koster, G. Rijnders, H. Hilgenkamp, and D. H. A. Blank, Adv. Mater. 21, 1665 (2009).

[30] C. Bell, S. Harashima, Y. Kozuka, M. Kim, B. G. Kim, Y. Hikita, and H. Y. Hwang, Phys. Rev. Lett. 103, 226802 (2009). 
[31] M. Ben Shalom, A. Ron, A. Palevski, and Y. Dagan, Phys. Rev. Lett. 105, 206401 (2010).

[32] M. Huijben, G. Koster, M. Kruize, S. Wenderich, J. Verbeeck, S. Bals, E. Slooten, B. Shi, H. Molegraaf, J. Kleibeuker, S. van Aert, J. Goedkoop, A. Brinkman, D. Blank, M. Golden, G. van Tendeloo, H. Hilgenkamp, and G. Rijnders, Adv. Funct. Mater. 23, 5240 (2013).

[33] J. S. Kim, S. S. A. Seo, M. F. Chisholm, R. K. Kremer, H.-U. Habermeier, B. Keimer, and H. N. Lee, Phys. Rev. B 82, 201407(R) (2010).

[34] J. Krupka, R. Geyer, M. Kuhn, and J. Hinken, IEEE Trans. Microwave Theory Tech. 42, 1886 (1994).

[35] T. Sakudo and H. Unoki, Phys. Rev. Lett. 26, 851 (1971).

[36] W. Siemons, M. Huijben, G. Rijnders, D. H. A. Blank, T. H. Geballe, M. R. Beasley, and G. Koster, Phys. Rev. B 81, 241308(R) (2010).
[37] A. E. M. Smink, J. C. de Boer, M. P. Stehno, A. Brinkman, W. G. van der Wiel, and H. Hilgenkamp, Phys. Rev. Lett. 118, 106401 (2017).

[38] M. Diez, A. M. R. V. L. Monteiro, G. Mattoni, E. Cobanera, T. Hyart, E. Mulazimoglu, N. Bovenzi, C. W. J. Beenakker, and A. D. Caviglia, Phys. Rev. Lett. 115, 016803 (2015).

[39] M. W. Wu, J. H. Jiang, and M. Q. Weng, Phys. Rep. 493, 61 (2010).

[40] P. Boross, B. Dora, A. Kiss, and F. Simon, Sci. Rep. 3, 3233 (2013).

[41] S. Maekawa and H. Fukuyama, J. Phys. Soc. Jpn. 50, 2516 (1981).

[42] J. Biscaras, N. Bergeal, A. Kushwaha, T. Wolf, A. Rastogi, R. Budhani, and J. Lesueur, Nat. Commun. 1, 89 (2010).

[43] L. Mattheiss, Phys. Rev. B 6, 4740 (1972). 